\title{
SPRACHLICHE MITTEL DER AUFMERKSAMKEITSLENKUNG AM BEISPIEL DEUTSCHER HOROSKOPTEXTE
}

\begin{abstract}
AвSTRACT: The linguistic means used to draw the reader's attention on the example of press horoscopes in German

Press horoscopes are short informative and persuasive texts addressed to a seemingly individual reader, who skims through the text in order to match the content and intention of press horoscopes to his or her own situation. The article deals with the selected linguistic means used in German press horoscopes to draw the reader's attention to certain text elements. The analysed data consists of extracts from press horoscopes in German weekly magazines for women (Bild der Frau, Freundin). The aim of the paper is to investigate different linguistic means (e.g. syntactic, lexical and typographical ones) used to draw the reader's attention in order to elaborate their preliminary subclassification.
\end{abstract}

KEYWORDS: press horoscope, emphasis, German syntax and pragmatics, emphasizing lexemes, punctuation

\section{Zielsetzung}

Der Beitrag setzt sich zum Ziel, am Beispiel von Pressehoroskopen sprachliche Mittel zu untersuchen, die die Aufmerksamkeit des Lesers auf bestimmte Textelemente lenken, um diese anschließend vorläufig zu klassifizieren. Aufmerksamkeitslenkung setze ich in meinem Text mit Auffälligmachung gleich, d. h., ich betrachte sie als ,kommunikatives Vorgehen, dem die Absicht zugrunde liegt, Wichtiges von dem unwichtigen Informationshintergrund abzuheben“"(Cirko, 2013, S. 55). Da die Gewinnung der Aufmerksamkeit des Lesers durch den Autor auf allen Ebenen des Sprachsystems erfolgen kann, werde ich die zu untersuchenden Mittel in syntaktisch-topologische, lexikalische und graphemische Mittel untergliedern.

Die empirische Basis der von mir durchgeführten Analyse bilden Belege aus zwei Monatshoroskopen und 27 Wochenhoroskopen (darunter drei Single- und drei Liebeshoroskope) aus den Frauenzeitschriften Bild der Frau (BdF) und Freundin (F). Die

Agnieszka Poźlewicz - Uniwersytet im. Adama Mickiewicza, Poznań, apoz@amu.edu.pl 
analysierten Exzerpte sind zehn Print-Ausgaben von Bild der Frau, zwei Print-Ausgaben und 15 Online-Ausgaben von Freundin vom August und September 2014 und November und Dezember 2015 entnommen.

\section{Textsorte Pressehoroskop}

Die Textsorte Pressehoroskop gilt als „Vertreter medienspezifischer Kleintextsorten mit deutlich unterhaltendem Charakter und stark ausgeprägter Konventionalisierung sowohl der Inhalte als auch ihrer sprachlichen Realisierung" (Schatte, 2010, S. 155). Diese zeichnet sich durch Mehrfachadressiertheit aus, da sie „den schwierigen Spagat bewältigen muss zwischen Ansprache eines Massenpublikums und gleichzeitiger Befriedigung individueller Leserbedürfnisse“" (Bachmann-Stein, 2004, S. 74), was Konsequenzen für ihre Textstruktur, aber auch für ihre sprachliche Gestaltung hat.

Heinemann rechnet Pressehoroskope zu den „Textsorten der Alltagskommunikation i. w. S. “, d. h. „zu Schrifttextsorten, die zwar das Alltagsleben von einzelnen Individuen und Gruppen mitbestimmen, aber - quasi von außen kommend, über Medien und Institutionen vermittelt - nur rezipiert und verarbeitet werden" (Heinemann, 2000, S. 609, Hervorhebung im Original). Als solche sind sie ,ein ideales Beispiel für die Rezeptionsweise des selektiven Lesens" (Fandrych \& Thurmair, 2011, S. 175), da sich ihre Leser nur auf „das eigene Horoskop (d. h. den Teiltext, der sich auf das eigene Sternzeichen bezieht) [...], das des Partners / der Partnerin und manchmal auch noch das Horoskop von Menschen, die ihnen nahestehen“" (Fandrych \& Thurmair, 2011, ebd.) konzentrieren.

In der Analyse werde ich der Frage nachgehen, wie die Autoren der immer mehrfach adressierten und kurzen Horoskoptexte die Aufmerksamkeit ihrer flüchtig lesenden Leser zu lenken suchen.

\section{Analyse des gesammelten Materials}

\subsection{Syntaktisch-topologische Mittel}

In der Analyse der gesammelten Belege gehe ich von den syntaktisch-topologischen Mitteln aus, da diese - als Pendant zu den für die gesprochene Sprache charakteristischen prosodischen Mitteln - ,die mit Abstand wichtigste Hervorhebungsart“ (Cirko, 2013, S. 57) sind. Zu ihnen gehört in erster Linie die Herausstellung, d. h. „syntaktisches Satzumformungsverfahren, in dessen Zuge ein Element nach außerhalb des Satzverbandes verschoben und somit informativ hervorgehoben wird" (Cirko, 2013, S. 55). $\mathrm{Zu}$ unterscheiden ist unter diesen einerseits die Linksversetzung, bei der ein Satzelement der übrigen Satzstruktur vorangestellt wird, wie in folgenden Belegen: 
Neue Job-Idee? Jetzt könnte sie zünden! (BdF 28/2015, Waage)

Gute Intuition - folgen Sie ihr! (BdF 31/2015, Jungfrau)

Miete, Handy, Auto - das schlägt zu Buche. (F online, Sept. 2014, Waage)

Herzklopfen und Wimperklimpern, Händchenhalten und lange Blicke - all das belebt die Liebe in dieser Phase und lässt in Ihrer Gegenwart die schönsten Geigen erklingen. (F online, Dez. 2015, Krebs)

Neuer Mut, neue Sicht der Dinge, Selbstvertrauen - alles klappt! (BdF 37/2015, Fische)

Die Linksversetzung dient somit „, der vorausorientierenden Aufmerksamkeitssteuerung, um einen thematischen Aspekt [...] einzuleiten“ (Bachmann-Stein, 2004, S. 240). Das oft graphemisch mit einem Gedankenstrich isolierte herausgestellte Element wird dabei mit einem Personal- (sie, ihr), Demonstrativ- (das) oder Indefinitpronomen (alles) wieder aufgenommen. Neben der pronominalen Wiederaufnahme ist allerdings auch eine nominale möglich, mit der das Linksversetzte im Satz durch eine Nominalphrase summiert oder (wertend) erklärt wird, wie in folgenden Belegen:

Baum schmücken, Weihnachtseinkäufe schleppen, Treppen rennen - der Sport kommt in dieser Woche eigentlich ganz von selbst. (F online, Dez. 2015, Widder)

Ob Grillparty, Radtour oder Badetag am Baggersee - Sie genießen einfach diese schöne Phase. (F online, Aug. 2014, Skorpion)

$\mathrm{Zu}$ den oben angeführten Belegen ist anzumerken, dass in ihnen durch Linksversetzung Akkumulationen zweier oder dreier Elemente mit nah beieinander liegenden Bedeutungen herausgestellt werden. Diese Zweier- und Dreierfiguren sind für Pressehoroskope typische Formulierungsmuster, auf deren Funktion ich unten detailliert eingehe.

In dem von mir ausgewerteten Material finden sich außerdem Belege für die Herausstellung am rechten Satzrand. Erstens ist hier die Rechtsversetzung zu erwähnen, wie in folgenden Belegen:

Venus und Pluto verheißen das, wovon alle träumen: Eine leidenschaftliche Liebe auf den ersten Blick, die so tief geht, dass sie für immer sein könnte. (F online, Dez. 2015, Krebs)

Bei der Umsetzung Ihrer beruflichen Pläne ist jetzt vor allem eins wichtig: Partnerschaft. (F online, Sept. 2014, Fische)

Sie können sich jetzt Ihren wichtigsten Themen widmen: der Familie, dem Heim und Ihrer Gefühlswelt. (F 21/2014, Krebs)

Die ursprüngliche Stelle des nach rechts herausgestellten Elements ist in den Belegen durch eine Proform wie etwa das, die Kardinalzahl eins oder durch eine allgemeine und summierende Nominalphrase (Ihre wichtigsten Themen) markiert. Das Rechtsversetzte wird graphemisch mit einem Doppelpunkt abgegrenzt, der „die Aufmerksamkeit des Lesers katadeiktisch auf das [lenkt], was ihm unmittelbar folgt" (Zifonun \& Hoffmann \& Strecker, 1997, S. 299).

Zweitens ist die Ausklammerung zu nennen, mit der die besonders hervorzuhebenden Satzglieder der rechten Satzklammer nachgestellt werden. In dem von mir 
analysierten Material sind die ausgeklammerten Elemente graphemisch mit einem Gedankenstrich isoliert, was folgende Belege illustrieren:

Lassen Sie los - Ihre Zweifel und Ängste! (BdF 50/2015, Steinbock)

Lästige Alltagspflichten bremsen Sie aus - noch! (BdF 33/2015, Waage)

Dennoch sollten Sie keine allzu hohen Erwartungen hegen, sonst sind Sie hinterher nur enttäuscht und das völlig unnötig. (F online, Aug. 2014, Fische)

Feurig! Beim Flirten nicht zu bremsen - und in der Liebe erst recht nicht... (BdF 37/2015, Wassermann)

Es wird also nicht langweilig - auch nicht im Freundeskreis! (F online, Aug. 2014, Zwillinge)

Drittens dient der Aufmerksamkeitssteuerung der Nachtrag, bei dem bestimmte Elemente ,aus ihrer üblichen Position aus dem Satzrahmen verlagert [werden] und [...] dadurch stärkere Akzentuierung [erfahren]“" (Furthmann, 2006, S. 428). Oft dient der Nachtrag auch der Präzisierung oder Erklärung des vorher Gesagten wie in folgenden Belegen:

Charmant wickeln Sie alle um den Finger. Auch den Partner. (BdF 32/2015, Krebs)

Grundsätzlich können Sie jetzt in allen Bereichen Ihres Lebens erfolgreich sein, privat wie beruflich. (F 16/2014, Widder)

Ihre Auswahlkriterien sind ganz schön anspruchsvoll und trotzdem wimmelt es nur so vor interessanten Kandidaten, zumindest auf den ersten Blick. (F online, Aug. 2014, Löwe)

In dieser Woche bietet Mars noch einmal gute Gelegenheiten, um sich gesundheitlichen Fragen zu widmen - vor allem solchen, die mit Bewegung zu tun haben. (F online, Sept. 2014, Zwillinge)

In Ihre Beziehung zieht im Dezember viel Leidenschaft ein. Das kann sich auf verschiedene Arten äußern - auch auf unangenehme. (Monatshoroskop, F online, Dez. 2015, Wassermann)

Pure Harmonie steht in den Sternen - und zwar eine Harmonie, die sehr angenehm und natürlich entsteht. (F online, Dez. 2015, Zwillinge)

Zum letzten der oben angeführten Belege ist anzumerken, dass in ihm der Nachtrag durch den Erläuterungen oder Korrekturen einleitenden präzisierenden Konjunktor und zwar signalisiert ist (vgl. Rytel-Schwarz et al., 2012, S. 170).

Als ein weiteres syntaktisches Mittel der Aufmerksamkeitslenkung gilt darüber hinaus der für Horoskope typische Hack-Stil ,in Form von Infinitiv-, Partizipialbzw. Adjektivkonstruktionen und zahlreichen Einwortformulierungen" (Schatte \& Poźlewicz, 2015, S. 377). Bachmann-Stein (2004, S. 238) weist zudem darauf hin, dass ,die Isolierung der einzelnen Äußerungen [...] der inhaltlichen Verstärkung und Hervorhebung [dient]". Folgende Belege von Einwortäußerungen und Routineformeln mögen dies illustrieren. Sie fungieren als emotionalisierende Kommentare, die die Aufmerksamkeit des Lesers auf das Voran- oder Nachgestellte lenken:

Aus Angst, zu viel von sich preiszugeben, verschweigen Sie Wünsche. Falsch! (BdF 44/2015, Fische) Jemand vertraut Ihnen ein Geheimnis an. Diskretion! (BdF 32/2015, Waage)

Im Job öffnen sich neue Türen. Erfolg! (BdF 50/2015, Waage) 
Sie geben alles, um Ihren Lieben eine traumhafte Weihnacht auszurichten. Mit Erfolg! (Monatshoroskop, BdF 50/2015, Krebs)

Sie nutzen Kritik, um dazuzulernen. Bravo! (BdF 32/2015, Krebs)

Gute Nachrichten! Ihnen fällt ein Stein vom Herzen. (BdF 32/2015, Fische)

Veränderungen im Job? Keine Sorge - die werden vorteilhaft für Sie! (BdF 32/2015, Fische)

Diese „vielfältigen meist kurzen syntaktischen Strukturen dienen der Emphase, vermitteln Dynamik und Lebendigkeit und verstärken den Eindruck mündlicher, scheinbar persönlicher, unmittelbarer und spontaner Sprache“ (Furthmann, 2006, S. 429).

\subsection{Lexikalische Mittel}

$\mathrm{Zu}$ den die Aufmerksamkeit des Lesers steuernden lexikalischen Mitteln gehören die oben erwähnten Akkumulationen bedeutungsnaher Elemente. „Ihre Häufung [erhöht] die expressive Wirkung [...] und [verleiht] der ganzen Äußerung mehr Emotionalität" (Schatte \& Poźlewicz, 2015, S. 367). Ihre Funktion besteht darin, „einen Sachverhalt besonders hervorzuheben oder einen Themenbereich auszuweiten" (Furthmann, 2006, S. 388).

In dem von mir ausgewerteten Material finden sich einerseits Akkumulationen von zwei, drei oder sogar vier Adjektiven im Positiv oder Komparativ, die gelegentlich mit Gradattributen wie sehr und besonders intensiviert sind. Die Verwendung des Komparativs und der intensivierenden Adverbien und Partikeln erhöht die für Horoskoptexte charakteristische Vagheit des Gesagten zusätzlich durch scheinbare Präzisierungen, die „stark vom individuellen Rezipientenkontext abhängig sind” (Furthmann, 2006, S. 326). Bei den Akkumulationen handelt es sich vorwiegend um ,positiv wertende Adjektive, meistens aus der Gruppe der sog. Hochwertadjektive“ (Schatte, 2010, S. 158). Die mit dem additiven Konjunktor $u n d$ verbundenen oder seltener asyndetisch gehäuften Adjektive fungieren meistens als Prädikativ, stehen aber oft in den für den Hack-Stil der Horoskoptexte charakteristischen Kürzungen, die durch das Weglassen des Kopulaverbs (Sie sind... bzw. Sie werden ...) entstehen, was folgende Belege illustrieren:

Tüchtig und durchsetzungsfähig, beliebt und bewundert. (BdF 50/2015, Waage)

Charmant, sehr sinnlich, liebevoll und zärtlich. (BdF 28/2015, Krebs)

Energiegeladen, optimistisch und sehr charmant mit Merkur, Venus, Mars und Uranus. (Monatshoroskop, BdF 50/2015, Wassermann)

Sehr anlehnungsbedürftig, einfühlsam und verführbar. (BdF 50/2015, Krebs)

Sie sind hilfsbereit, zuverlässig und einfühlsam. (BdF 50/2015, Fische)

Das wird eine Liebe zum Verlieben: Romantischer, sinnlicher und schöner kann es unter der aktuellen Venus-Neptun-Verbindung nicht werden. (F online, Dez. 2015, Krebs)

Sie sind kontaktfreudiger, offener, unterhaltsamer. (BdF 50/2015, Steinbock)

Im von mir analysierten Material finden sich allerdings auch Belege für adjektivische Akkumulationen von Attributen in Nominalphrasen: 
Es kann nicht schaden, auch im Dezember auf eine besonders zuvorkommende, verbindliche und höfliche Kommunikation zu achten. (Monatshoroskop, F online, Dez. 2015, Steinbock)

Singles könnten jetzt dem Menschen begegnen, der das Schicksalsblatt für sie neu mischt. Eine weitreichende, wichtige und schöne Phase! (F online, Dez. 2015, Fische)

Neben den Adjektiven werden auch Nomina in Dreier-, seltener in Vierer- oder sogar Fünfergruppen syndetisch mit dem additiven Konjunktor und oder asyndetisch verwendet. „Die einander gereihten Begriffe beziehen sich dabei meist auf einen genannten oder nicht genannten Oberbegriff" (Furthmann, 2006, S. 388):

Schönste Tage für Dates, Flirts, Kunst, Kultur, Vergnügen. (BdF 44/2015, Zwillinge)

Super für Kontakte, Liebe, Job und Wohnungssuche. (BdF 34/2015, Waage)

An Schwung, Elan und Wohlbefinden mangelt es Ihnen daher nicht, und so machen Job, Haushalt und Fitnesstraining kaum Schwierigkeiten. (Monatshoroskop, F online, Dez. 2015, Wassermann) Beliebt in der Familie, bei Freunden und Bekannten. (Monatshoroskop, BdF 50/2015, Schütze) Lust auf Abenteuer, Liebe und Erfolg! (BdF 37/2015, Schütze)

Unwiderstehliche Mischung aus Charme, Temperament und Stärke. (Monatshoroskop, BdF 50/2015, Widder)

Geschickt lenken Sie Kollegen, Partner, Familie - und keiner merkt es! (BdF 31/2015, Jungfrau)

Die im reinen oder im Präpositionalkasus stehenden Nomina werden gelegentlich mit Adjektiven, seltener mit Präpositionalphrasen oder mit dem Quantifikator viel attribuiert, was folgende Belege illustrieren:

Schöne Erfolge, großes Manipulationsgeschick, heiße Flirts. (Monatshoroskop, BdF 50/2015, Steinbock)

Glückssträhne im Job. Erfolge. Gute Geschäfte, Glück bei Prüfungen. (Monatshoroskop, BdF 50/2015, Fische)

Scharfer Verstand, viel Geduld und Durchhaltevermögen. (Monatshoroskop, BdF 50/2015, Jungfrau) Viele Bewunderer, Komplimente und Einladungen. (Monatshoroskop, BdF 50/2015, Wassermann) Das wird eine Woche der Feste, der Geselligkeiten und der gehobenen Stimmung. (F online, Dez. 2015, Löwe)

Kleine Gesten, Blicke und Berührungen sind derzeit wichtiger und vielsagender als lange Gespräche. (F online, Dez. 2015, Waage)

Der Skorpion-Mars bringt Energie, Vitalität und prima Laune. (F online, Sept. 2014, Jungfrau)

Die nominalen Akkumulationen stehen typischerweise in verkürzten adjektivischen Phrasen, die den Hack-Stil der Horoskoptexte bestimmen und nach dem Muster positiv wertendes Adjektiv (fantastisch, günstig, ideal, optimal, super) für + nominale Akkumulation gebildet sind. Solche Konstruktionen

[...] benennen Gegebenheiten oder empfohlene Verhaltensweisen, enthalten aber auch aufbauende Feststellungen über Zustände und Eigenschaften und sind meist als Reduzierungen einer im Horoskopkontext sich erübrigenden einleitenden Voraussage zu verstehen. (Schatte \& Poźlewicz, 2015, S. 377) 
Fantastisch für Reisebuchung, Ausflüge, Familientreffen. (Monatshoroskop, BdF 50/2015, Wassermann)

Günstig für Inneneinrichtung, Renovierung, Möbelkauf. (BdF 35/2015, Stier)

Ideal für Prüfungen, Geschäfte, Reisen. (BdF 37/2015, Waage)

Optimal für Reise, Geschäfte, Vorstellungsgespräche. (BdF 28/2015, Steinbock)

Super für Geselligkeit, Reisestart und Urlaub. (Monatshoroskop, BdF 50/2015, Zwillinge)

Viel seltener werden Verben gehäuft, wie etwa in folgenden Belegen:

Mit Ihnen steht und fällt einfach jede Feier. Gehen Sie hin, machen Sie mit, feiern Sie! (F online, Dez. 2015, Zwillinge)

Sinnlich. Sie bezaubern, faszinieren, verführen... (BdF 37/2015, Waage)

Wollen Sie umbauen, renovieren, gärtnern? Freunde helfen jetzt. (BdF 28/2015, Krebs)

Ein weiteres lexikalisches Mittel sind Figuren der Wiederholung, ,welche der nachdrücklichen Hervorhebung und Verstärkung bestimmter Aussagen und dem Wecken und Fördern von Aufmerksamkeit beim Leser dienen“ (Furthmann, 2006, S. 387). Im von mir ausgewerteten Material finden sich Belege für die Geminatio, d. h. die „,unmittelbare Wiederholung eines Ausdrucks mit intensivierender Wirkung" (Bußmann, 2002, S. 239), sowie für die Anapher, bei der ein Ausdruck ,am Beginn aufeinander folgender Sätze, Satzteile oder Textabschnitte“ (Bußmann, 2002, S. 79) wiederholt wird. Solche Konstruktionen wirken „eindringlich, rhythmisierend und damit einprägsam“ (Bußmann, 2002, ebd.):

Es wird scharf und sehr, sehr sinnlich! (F online, Dez. 2015, Skorpion)

Dürfte es etwas mehr sein? Mehr Geld, mehr Respekt von den Kollegen und Vorgesetzten? Kommt sofort! Jupiter und Sonne bringen Ihnen den Erfolg. (F online, Dez. 2015, Wassermann)

Wünschen Sie sich nicht, dass das Leben leichter wird - wünschen Sie sich, dass Sie stärker werden! (BdF 50/2015, Widder)

Venus und Neptun deuten auf einen kleinen, aber verbotenen Flirt hin. Das wird heiß, das wird kitzelnd - und das wird vor allem nervig. (F online, Dez. 2015, Widder)

Zu den lexikalischen Mitteln der Aufmerksamkeitslenkung gehören darüber hinaus expositive Operatorpartikeln, deren primäre semantische Leistung in der Hervorhebung ihrer Operanden besteht (vgl. Poźlewicz, 2011, S. 144). Im von mir analysierten Material dominieren die generell austauschbaren Partikeln besonders und vor allem, die ein Element aus einer Menge weiterer Elemente als vorrangig exponieren. Sie signalisieren Alternativen für das explizit Genannte, wodurch „die Voraussage weniger festgelegt [ist] und [...] somit individueller Interpretation [bedarf]“" (Schatte \& Poźlewicz, 2015, S. 372). Darüber hinaus wird damit ,zusätzlich [...] der Aspekt der Voraussage hervorgehoben, dem die Aufmerksamkeit des Rezipienten besonders gelten soll“" (Schatte \& Poźlewicz, 2015, S. 373). Die Analyse der gesammelten Belege ergibt, dass beide Partikeln oft parenthetische Einschübe oder Nachträge eröffnen und 
so der Präzisierung des Gesagten dienen. Das nachgetragene oder präzisierte Element ist graphemisch oft mit einem Gedankenstrich isoliert, wie in folgenden Belegen:

Besonders Anfang des Monats öffnen sich Ihnen neue Türen zum Erfolg. (Monatshoroskop, BdF 50/2015, Schütze)

Lassen Sie es nicht dazu kommen, besonders jetzt am Anfang nicht! (F online, Dez. 2015, Krebs)

Sehr flexibel - bes. beim Flirten. (BdF 31/2015, Waage)

Jupiter öffnet Sie für alles Schöne - bes. für die Liebe. (BdF 28/2015, Krebs)

Gegner - bes. im Job - freundlich ins Leere laufen lassen. (BdF 50/2015, Schütze)

Kluge Strategien - bes. bei Verhandlungen. (BdF 34/2015, Stier)

Nach außen sanft, aber wenn's drauf ankommt, setzen Sie sich durch - bes. in der Familie. (Monatshoroskop, BdF 50/2015, Steinbock)

Endlich tut sich etwas und dieses Etwas ist vor allem für Singles mehr als interessant. (F online, Aug. 2014, Zwillinge)

Sind Sie liiert, dann peppen Sie mit Ihrem Einfallsreichtum die Beziehung auf, vor allem am Wochenende zum kreativen Wassermann-Mond! (F online, Sept. 2014, Waage)

Entwickeln Sie deshalb einen Bewegungsplan dagegen - vor allem fürs Wochenende - der Sie und auch den Schatz oder die Kinder ein bisschen mehr an die frische Luft bringt. (F online, Dez. 2015, Skorpion) In Beziehungen kommt man sich wieder näher und Singles flirten, was das Zeug hält. Nicht unwahrscheinlich, dass Sie nun die Nummer Ihres Traumpartners bekommen - vor allem von Montag bis Mittwoch, denn dann flüstert Ihnen der Mond zu, wem Sie vertrauen können. (F online, Aug. 2014, Skorpion)

Zur Operatorpartikel besonders ist anzumerken, dass sie bevorzugt in syntaktisch gekürzten Strukturen auftritt.

Eine ähnliche Funktion weist die Operatorpartikel sogar auf, die ein Element ,als etwas unerwartet Realisierbares/Realisiertes“ (Rytel-Schwarz et al., 2012, S. 37) hervorhebt. Das veranschaulichen folgende Belege:

Ihre tollen Ergebnisse können sogar ein höheres Einkommen zur Folge haben. (Monatshoroskop, F online, Dez. 2015, Stier)

Selbständige können neue Aufträge einheimsen und Angestellte sogar eine Beförderung. (F online, Dez. 2015, Skorpion)

Superstark und ehrgeizig ertragen Sie sogar Belastungen, an denen andere zerbrechen würden. (Monatshoroskop, BdF 50/2015, Skorpion)

Dazu arbeitet Ihr Geist auf Hochtouren und Sie finden jetzt sogar für besonders knifflige Probleme schnell eine Lösung. (F online, Aug. 2014, Skorpion)

Ähnlich wie besonders und vor allem kann auch die Partikel sogar nachträgliche Präzisierungen signalisieren. Auch hier wird der Nachtrag u. U. mit einem Gedankenstrich graphemisch isoliert, um die Aufmerksamkeit des Lesers zu lenken. Das zeigen folgende Belege:

Sie scheinen einen Gönner zu haben, vielleicht sogar jemanden, von dem Sie gar nichts ahnen. (F online, Dez. 2015, Wassermann) 
Sie werden alles erfolgreich meistern und können damit Ihre Position in der Firma - und vielleicht sogar finanziell - verbessern. (F online, Dez. 2015, Skorpion)

Das gibt ordentlich Pluspunkte beim Chef und prädestiniert Sie für künftige Aufgaben - oder sogar eine Beförderung. (F online, Sept. 2014, Skorpion)

Es knistert - sogar im Freundeskreis. (BdF 34/2015, Schütze)

Auch die redebezogenen und eher für die gesprochene Sprache typischen Abtönungspartikeln können die Aufmerksamkeit des Lesers steuern, indem sie „der Hervorhebung, der Verstärkung und Abschwächung von Sprechakten (z. B. von Aufforderungen) [dienen]“ (Schwitalla, 2012, S. 154). Gleichzeitig erwecken sie „den Eindruck der Alltagsnähe, Mündlichkeit und Expressivität" (Schatte \& Poźlewicz, 2015, S. 374). Im ausgewerteten Material dominieren die Abtönungspartikeln bloß und doch in den im Horoskop erteilten Aufforderungen, Ratschlägen und Empfehlungen, um diese dringlicher erscheinen zu lassen bzw. ihnen stärkeren Nachdruck zu verleihen (vgl. Duch-Adamczyk, 2012, S. 110 f.):

Ein Verflossener versucht wieder anzubandeln. Bloß nicht schwach werden! (BdF 34/2015, Löwe)

Bloß jetzt keinen Familienstreit anzetteln! (BdF 30/2015, Waage)

Sich und andere bloß nicht überfordern. (BdF 50/2015, Skorpion)

Machen Sie doch noch schnell einen spannenden Kurs oder eine Last-Minute-Weiterbildung! (F online, Dez. 2015, Wassermann)

Übernehmen Sie doch dieses Jahr die Organisation der Silvesterfeier oder des Weihnachtsfestes für sich und Ihre Leute! (F online, Dez. 2015, Fische)

Laden Sie doch eventuell selbst ein paar Leute ein, und deren Freunde, sodass Sie nun noch mal neue Menschen kennenlernen können. (F online, Dez. 2015, Widder)

Eigentlich wünschen Sie sich vielmehr innere und auch äußere Ruhe. Versuchen Sie doch, das zu bewerkstelligen. (F online, Dez. 2015, Wassermann)

Die Partikel bloß scheint im analysierten Material vor allem in minimalisierten unpersönlichen Infinitivkonstruktionen zu stehen, die Partikel doch indessen eher in den unverkürzten Aufforderungssätzen.

Als weiteres lexikalisches Mittel der Aufmerksamkeitslenkung gelten die Interjektionen, wie hey, oje oder juchuh, die ,dem Ausdruck spontaner, reaktiver Emotionen oder Bewertungen [dienen]“" (Eisenberg et al., 2009, S. 597). Ähnlich wie die Abtönungspartikeln sollen sie „eine vertraut-persönliche Gesprächssituation nachbilden“(Furthmann, 2006, S. 426). Durch die so hergestellte Dialogizität kann man „den Kontakt zum Leser unmittelbar herstellen und aufrechterhalten [...]. Man suggeriert Vertrautheit des Sprechers mit dem individuellen Adressaten, Interesse am persönlichen Leben des Lesers sowie Spontaneität“ (Furthmann, 2006, S. 176). Wie die unten angeführten Belege zeigen, sind die Interjektionen syntaktisch nicht integriert und stehen als autonome Äußerungen satzassoziiert vor einem vollständigen Satz als wertender Kommentar zu diesem (vgl. Eisenberg et al., 2009, S. 597): 
Hey, Sie Party-Löwe! Ob Weihnachtsfeier oder vorgezogener Silvesterempfang, Sie sind unter Sonne und Uranus immer ganz vorne mit dabei. (F online, Dez. 2015, Löwe)

Es herrschen Romantik und Eintracht, man ist sich sehr nah und zweifelt nicht an der Partnerschaft. Hach, wie schön kann die Liebe sein... (F online, Dez. 2015, Steinbock)

Oje, in Sachen Gesundheit machen es Ihnen die Sterne nicht leicht. (F online, Nov. 2015, Zwillinge) Venus und Neptun begünstigen Ihr Liebesglück: Sie könnten Ihren Traumpartner treffen. Juchuh! Endlich, werden Sie denken, und er genauso. (F online, Dez. 2015, Zwillinge)

O weh, Venus und Neptun geben einen deutlichen Hinweis, dass die Liebe jetzt nicht ganz ungetrübt ist. (F online, Dez. 2015, Schütze)

Wow, dank Merkur in Verbindung mit Neptun und Pluto sind Sie geistig auf Zack und zugleich ausgesprochen kreativ. (F online, Dez. 2015, Steinbock)

Hinzuweisen ist außerdem auf die pragmatischen Phraseologismen Achtung und Vorsicht, die als vorangestellte Kommentare die Aufmerksamkeit des Lesers auf die Folgeäußerung lenken, um ihn zu warnen oder zu mahnen. Dies illustrieren folgende Belege, in denen diese Aufmerksamkeitssignale (vgl. Bachmann-Stein, 2004, S. 237) als syntaktisch selbständige Äußerungen graphemisch isoliert sind:

Achtung, vorm Fest der Liebe kann's stressig werden. (Monatshoroskop, BdF 50/2015)

Achtung: wer Sie jetzt anpumpt, hört nicht mehr damit auf. (BdF 33/2015, Fische)

Vorsicht - aggressive Rivalin! (BdF 33/2015, Skorpion)

Vorsicht, Erkältungs-Gefahr! (Monatshoroskop, BdF 50/2015, Fische)

Vorsicht, nicht aushorchen lassen! (BdF 50/2015, Zwillinge)

Vorsicht: jemand im Job fädelt Intrigen ein! (Monatshoroskop, BdF 50/2015, Zwillinge)

Zugleich sind Sie aber auch sehr verträumt. Vorsicht, dass Sie unter Merkur und Neptun nicht etwas vergessen. (F online, Dez. 2015, Waage)

Pragmatische Phraseologismen können u. U. modifiziert werden, wie etwa mit den adversativen Konjunktoren aber und doch, mit direkter Anrede einer Adressatengruppe (Steinböcke) oder mit präzisierenden Attributen wie jetzt, im Job:

Doch Achtung, wer es jetzt auf die große Liebe für ewig abgesehen hat, hat Pech. (F online, Nov. 2015, Widder)

Achtung, Steinböcke! In der Liebe warten diese Woche Herausforderungen auf Sie. (F online, Nov. 2015, Steinbock)

Singles: Auf einer Feier gesteht Ihnen jemand seine Zuneigung. Aber Vorsicht, er könnte noch gebunden sein. (Monatshoroskop, BdF 50/2015, Stier)

Jetzt Vorsicht im Job: Ein besserwisserischer Ehrgeizling versucht sich in den Vordergrund zu drängen. Deutlich von ihm abgrenzen. (Monatshoroskop, BdF 50/2015, Waage)

\subsection{Graphemische Mittel}

Abschließend möchte ich noch auf die graphemischen Mittel der Aufmerksamkeitslenkung eingehen, die die oben beschriebenen syntaktisch-topologischen und lexikali- 
schen Mittel unterstützen. Als erstes unter diesen ist die typographische Gestaltung des Textes zu nennen: Fettdruck, Wechsel der Schriftart oder -größe, Versalien, Kursive oder Schriftfarben ,,dienen [...] dazu, beim Leser Aufmerksamkeit zu wecken, ihm die Orientierung zu erleichtern und den Horoskop-Komplex wie auch das Einzelhoroskop selbst zu gliedern und übersichtlicher zu machen“" (Bachmann-Stein, 2004, S. 100). In den von mir analysierten Zeitschriften dominiert in dieser Funktion der Fettdruck wie in folgenden Belegen:

Glückspilze des Monats: Heiße Flirts für Krebse, Löwen, Skorpione, Waagen, Schützen, Steinböcke und Fische. (Monatshoroskop, BdF 50/2015, Hervorhebung im Original)

Der Neumond in Ihrem Zeichen küsst Sie wach wie aus einem Dornröschenschlaf, und Sie haben das Gefühl, wieder sehr präsent zu sein. (F 21/2014, Waage, Hervorhebung im Original)

Zweitens kann die Zeichensetzung die Aufmerksamkeit des Lesers steuern, wie etwa dreifaches Fragezeichen oder die Kombination aus Frage- und Ausrufezeichen in folgenden Belegen, die zugleich emotionale Anteilnahme des Horoskopautors suggerieren:

Entscheidungs-Schwierigkeiten: auf welche Silvester-Party sollen Sie gehen??? (Monatshoroskop, BdF 50/2015, Zwillinge)

Gewagte Flirts im Urlaub?! Das gibt Stress mit dem Partner... (BdF 35/2015, Waage)

Liebe: Herz in Flammen?! Singles: Neuer Partner? Dann wird's romantisch. (BdF 37/2015, Widder)

Auch die graphemische Isolierung von Satzgliedern bzw. Teilsätzen mit Gedankenstrich oder Doppelpunkt, auf die oben mehrmals hingewiesen wurde, kann der Aufmerksamkeit des Lesers aufhelfen, weil dadurch ,ein anderes Tonmuster und damit eine Gewichtung beider Teile erreicht [wird]“" (Sandig, 2006, S. 230). So signalisiert der Gedankenstrich ,den Übergang zu einer besonders hervorgehobenen - oft inhaltlich unerwarteten - Konstituente“ (Zifonun \& Hoffmann \& Strecker, 1997, S. 301) wie etwa in:

Sie nutzen jede Chance - im Job wie privat. (BdF 31/2015, Schütze)

Ihre momentane Geselligkeit ist die beste Voraussetzung für heiße Flirts - und mehr! (F online, Sept. 2014, Stier)

Jemand begehrt Sie, ein anderer verwöhnt Sie - viele Flirt-Chancen! (BdF 30/2015, Stier)

Der Doppelpunkt indessen wird genutzt, ,um das darauf Folgende besonders hervorzuheben“ (Sandig, 2006, S. 230) und ,stellt zugleich eine spezifische Verknüpfungsbeziehung [...] zwischen Vorhergehendem und folgendem Ausdruck her" (Zifonun \& Hoffmann \& Strecker, 1997, S. 299). Im analysierten Material grenzt er einerseits vorweggenommene Kategorisierungen ab, die dem Leser durch die Ankündigung des Themenbereichs die Orientierung im Text erleichtern wie etwa in folgenden Belegen: 
Gefahr: Für Weihnachtseinkäufe überziehen manche ihr Ausgaben-Limit. (Monatshoroskop, BdF 50/2015, Widder)

Große Freude: Alte Freunde melden sich. (Monatshoroskop, BdF 50/2015, Schütze)

Heilende Aura: Wer Ihre Sympathie hat, tankt an Ihrer Seite neue Kraft. (Monatshoroskop, BdF 50/2015, Skorpion)

Andererseits kann der Doppelpunkt wegen des für Horoskoptexte typischen HackStils die Kopula ist ersetzen, um das Nachfolgende ,,abzutrennen und stärker hervorzuheben“ (Furthmann, 2006, S. 427), was folgende Belege veranschaulichen:

Gute Teamarbeiterin. Ihr zuverlässigster Verbündeter: ein Steinbock! (BdF 33/2015, Krebs)

Heiße Flirts. Ihr Favorit: ein Löwe! (BdF 31/2015, Waage)

Ihre beste Zeit im Job: Anfang des Monats mit Merkur. (Monatshoroskop, BdF 50/2015, Wassermann) Erfolg im Job. Ihre Stärken: uneingeschränkter Fleiß und diplomatisches Geschick. (Monatshoroskop, BdF 50/2015, Jungfrau)

\section{Zusammenfassung}

Die vorgenommene Analyse ausgewählter Horoskoptexte sollte zeigen, dass deren Autoren die Aufmerksamkeit des Lesers mit Mitteln verschiedener Ebenen des Sprachsystems lenken. Die oben betrachteten syntaktisch-topologischen, lexikalischen und graphemischen Mittel sind kombinierbar und stützen einander, was „den intendierten kommunikativen Effekt [verstärkt]" (Cirko, 2013, S. 72).

Aus der Analyse des gesammelten Materials ergibt sich darüber hinaus, dass einige der erfassten Mittel für Horoskoptexte typisch sind, so etwa die adjektivischen, nominalen oder verbalen Akkumulationen oder die reduzierten Infinitiv- oder Adjektivkonstruktionen, die den Hack-Stil der Pressehoroskope bestimmen. Es wurden zudem textsortenunabhängige Mittel der Aufmerksamkeitslenkung festgestellt, darunter die Herausstellung nach links oder rechts als syntaktisch-topologische Mittel und expositive Operatorpartikeln sowie pragmatische Phraseologismen als lexikalische Mittel. Deren spezifische Verwendung in anderen Textsorten bedarf allerdings entsprechend ausgerichteter weiterer Untersuchungen.

\section{Literatur}

Bachmann-Stein, A. (2004). Horoskope in der Presse. Ein Modell für holistische Textsortenanalysen und seine Anwendung. Frankfurt am Main: Peter Lang.

Bußmann, H. (Hrsg.). (2002). Lexikon der Sprachwissenschaft. Stuttgart: Kröner.

Cirko, L. (2013). Dem Gesagten Relief geben. In U. Engel (Hrsg.), Argumentieren. Sprechen im deutsch-polnischen Kontrast (S. 55-77). Wrocław-Dresden: Atut \& Neisse Verlag.

Duch-Adamczyk, J. (2012). Funktionsdistribution der Abtönungspartikeln des Deutschen und des Polnischen. Frankfurt am Main: Peter Lang. 
Eisenberg, P. \& Peters, J. \& Gallmann, P. \& Fabricius-Hansen, C. \& Nübling, D. \& Barz, I. \& Fritz, T.A. \& Fiehler, R. (2009). Duden. Die Grammatik. 8. Auflage. Mannheim-Wien-Zürich: Duden Verlag.

Fandrych, Ch. \& Thurmair, M. (2011). Textsorten im Deutschen. Linguistische Analysen aus sprachdidaktischer Sicht. Tübingen: Stauffenburg.

Furthmann, K. (2006). Die Sterne lügen nicht. Eine linguistische Analyse der Textsorte Pressehoroskop. Göttingen: V \& R Unipress.

Heinemann, M. (2000). Textsorten des Alltags. In K. Brinker \& G. Antos \& W. Heinemann \& S.F. Sager (Hrsg.), Text- und Gesprächslinguistik. Ein internationales Handbuch, 1. Halbband (S. 604-614). Berlin-New York: de Gruyter.

Poźlewicz, A. (2011). Syntax, Semantik und Pragmatik der Operatorpartikeln des Deutschen. Versuch einer Systematik. Frankfurt am Main: Peter Lang.

Rytel-Schwarz, D. \& Jurasz, A. \& Cirko, L. \& Engel, U. (2012). Deutsch-polnische kontrastive Grammatik. Band 4. Die unflektierten Wörter. Zweite, neu bearbeitete und ergänzte Auflage. Hildesheim-Zürich-New York: Georg Olms Verlag.

Sandig, B. (2006). Textstilistik des Deutschen. Berlin-New York: de Gruyter.

Schatte, Cz. (2010). Stilmittel in der Textsorte „Pressehoroskop“ im deutsch-polnischen Vergleich. In Z. Bilut-Homplewicz \& A. Mac \& M. Smykała \& I. Szwed (Hrsg.), Text und Stil (S. 155-163). Frankfurt am Main: Peter Lang.

Schatte, Cz. \& Poźlewicz, A. (2015). Thematisch-strukturelle und sprachliche Architektur der Textsorte Pressehoroskop in deutschen und polnischen Illustrierten. Kwartalnik Neofilologiczny, 62 (3), 359-385.

Schwitalla, J. (2012). Gesprochenes Deutsch. Eine Einführung. Berlin: Erich Schmidt Verlag.

Zifonun, G. \& Hoffmann, L. \& Strecker, B. (1997). Grammatik der deutschen Sprache. Berlin-New York: de Gruyter. 